\title{
Development of ICP-AES-based Methodology for the Determination of Trace Metallic Constituents in Zr-Nb Alloy
}

\author{
Sunita Pathak and Arijit Sengupta* \\ Radiochemistry Division, Bhabha Atomic Research Centre, \\ Mumbai, India
}

\section{INTRODUCTION}

Due to the advantageous properties such as low neutron absorption cross-section, high mechanical strength, good irradiation stability, and corrosion resistance, $\mathrm{Zr}$-based alloy materials are being extensively used as cladding and structural materials for nuclear fuel materials (1-5). Zircaloy-4 alloy has been used as cladding material for several years in the pressurized water reactor without any significant problem. To fulfill the energy demand, advanced Zr-based alloys are now being required for the drastic operating conditions such as higher burn-up, increased operating temperature, and high $\mathrm{pH}$ operation. Several new Zr-based alloy materials such as $\mathrm{Zr}-1$.0Nb$1.0 \mathrm{Sn}-0.1 \mathrm{Fe}, \mathrm{Zr}-1 \mathrm{Nb}-\mathrm{O}$, and $\mathrm{Zr}-0.1 \mathrm{Nb}-1.0 \mathrm{Sn}-0.27 \mathrm{Fe}-0.16 \mathrm{Cr}$ were synthesized, characterized, and tested in nuclear reactors (610 ), and the $\mathrm{Zr}-\mathrm{Nb}$ alloy showed very promising properties. The metallurgical properties and the performance of such nuclear materials depends largely on the concomitant metal ions present in the alloy (11-14). Therefore, a stringent chemical quality control is required to get its desired performance. In this manuscript, an ICP-AES-based method was developed for the determination of metallic constituents at the trace level in a $\mathrm{Zr}-\mathrm{Nb}$ matrix.

ICP-AES, being a simultaneously multi-elemental and reliable technique with desirable analytical performance including detection limit, sensitivity, linear dynamic range,

*Correspondingauthor.

E-mail: arijita@barc.gov.in

\begin{abstract}
A simple methodology was developed for the determination of trace metallic constituents (Ag, B, Ba, Bi, Ca, Cd, Ce, Ga, Gd, In, K, La, Li, Lu, Mg, Mn, Na, $\mathrm{Co}, \mathrm{Cr}, \mathrm{Cu}, \mathrm{Dy}, \mathrm{Eu}, \mathrm{Fe}, \mathrm{Nd}, \mathrm{Ni}$, $\mathrm{Pb}, \mathrm{Pr}, \mathrm{Sm}, \mathrm{Sr}, \mathrm{Tl}$, and $\mathrm{Zn}$ ) in $\mathrm{Zr}$ $\mathrm{Nb}$ alloy. The systematic study of the spectral interference of $\mathrm{Zr}$ as well as $\mathrm{Nb}$ was carried out on these analytes. This includes identification of interference-free lines, tolerance level, and correction factors associated with each line. The analytical performance of these lines including detection limit, sensitivity, and linear dynamic range was also investigated. Based on these two factors, the best line of each analyte was chosen. Chemical separation of the major matrix followed by the analysis of the raffinate was the strategy adopted in the development of the method. To optimize the chemical separation procedure, TBP, TOPO, and DHOA were used for preferential separation of the major matrix without loss of the analytes even at trace levels. The optimized method was validated using a synthetic sample, which revealed that five contacts of $1.1 \mathrm{M}$ TBP in dodecane was the ideal choice for separation and all of these analytes can be determined at the 1-mg L-1 level in a $\mathrm{Zr}-\mathrm{Nb}$ matrix with a RSD less than $5 \%$.
\end{abstract}

and precision, is routinely employed for the determination of trace metallic constituents in different nuclear materials (15-20). Due to the high excitation temperature of the plasma, more emission lines are available for the determination but some may lead to spectral interference. Mainly the nuclear material matrix elements are multi-electronic and have emission-rich spectra; hence, they are expected to show severe spectral interference while determining the analytes in these matrices especially at the trace level (21-25). Therefore, either incorporation of correction factors for spectral interference or chemical separation of the major matrix are the two strategies adopted in an ICP-AES-based methodology. The first option leads to a situation where the detection limit becomes poorer than the aqueous matrix, while the second option requires a suitable extractant which can preferentially separate the targeted emission-rich metal ion without extracting the analytes of interest even at the trace level. TBP (tri-nbutyl phosphate), DHOA (di-nhexyl octanamide), and TOPO (tri-n-octyl phosphate) were employed in the different stages of the nuclear fuel cycles, including its analytical application in trace metal assay of different nuclear material matrices (26-30). In the present case, a comparative study was carried out using these ligands to optimize the procedure: the aqueous feed acidity, the number of contacts required for separation of $\mathrm{Zr}$ as well as $\mathrm{Nb}$, and whether there is any loss of the analytes even at the trace level during the chemical separation of the major matrix.

\section{EXPERIMENTAL}

\section{Instrumentation}

The methodology was developed on a simultaneous multielemental inductively coupled plasma atomic emission spectrometer (Spectro-Arcos, Germany) with a high-performing charge-coupled device (CCD) as the detector. The 
optimized instrumental parameters are summarized elsewhere (31) and in Table I.

\section{Standard Solutions and Reagents}

Standard solutions for all of the analytes were prepared from CertiPUR $^{\circledR}$ ICP multi-standard solutions (E-Merck, Darmstadt, Germany) by proper dilution. Suprapur ${ }^{\circledR} \mathrm{HNO}_{3}$ (E-Merck) and quartz doubledistilled water were used throughout the investigation. Multi-point standardization was carried out using $0.5 \mathrm{M} \mathrm{HNO}_{3}$ as a lower standard and $0.05-1000 \mathrm{mg} / \mathrm{L}$ of the corresponding elemental standard as the higher standards for establishing calibration curves for all the analytes. All measurements were done in five replicates.TBP and TOPO were procured from Koch-

TABLE I

Specifications And Operating Conditions Of ICP-AES

\begin{tabular}{ll}
\hline Instrumental Specifications & \\
\hline Model No. & Arcos FH12 ICP-AES \\
Optical design & Paschen-Runge mounting, Circular design \\
Focal length & $750 \mathrm{~mm}$ \\
Grating & Holographic \\
Groove density & 1800 grooves/mm (1), 3600 grooves/mm (2) \\
Wavelength range & $130-800 \mathrm{~nm}$ \\
Entrance slit width & 15 microns \\
Resolution (FWHM) & 0.01 nm from $130-450 \mathrm{~nm}$ \\
& $0.02 \mathrm{~nm}$ from $450-800 \mathrm{~nm}$ \\
Thermal regulation & Controlled to $30 \pm 1{ }^{\circ} \mathrm{C}$ \\
Frequency & $27.12 \mathrm{MHz}$ \\
Pump & Dual channel peristaltic pump \\
Detector & Linear arrays of CCD (3648 pixels/array) \\
Nebulizer & Concentric nebulizer with cyclonic spray \\
ICP-torch & chamber \\
\hline Operating Conditions & Demountable, radial viewing \\
\hline Coolant flow & \\
Auxiliary flow & $16 \mathrm{~L} / \mathrm{min}$ \\
Total time of measurement & $30 \mathrm{~s}$ \\
Pump speed & $25 \mathrm{rpm}$ \\
RF power out put & $1.0 \mathrm{~kW}$ \\
\hline Input power & $230 \mathrm{~V}$ AC \\
\hline
\end{tabular}

Light Laboratories and Cytec Industries, Inc., USA. DHOA was synthesized by a previously reported method (32).

\section{Procedure}

During the spectral interference study, CertiPUR ${ }^{\circledR} \mathrm{Zr}$ and $\mathrm{Nb}$ solutions at different concentrations were fed to the plasma as the samples and the contribution in each analytical lines were monitored. The interference was found to vary linearly with the concentration of interfering ion and hence, the correction factors were calculated accordingly. The tolerance level of the interfering element was chosen as the maximum level of concentration of that element beyond which a particular line of analyte is interfered.

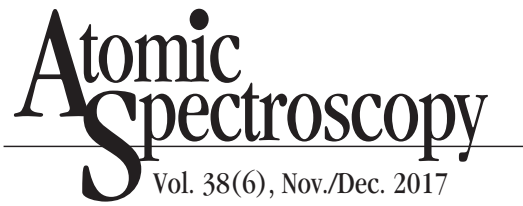

\section{Preparation of Synthetic Samples}

The synthetic samples were prepared by addition of suitable aliquots of the corresponding analytes from their individual CertiPUR ${ }^{\circledR}$ solutions. The acidity of the aqueous feed of the synthetic solution used for the ICP-AES analysis was kept at $3 \mathrm{M} \mathrm{HNO}_{3}$ as optimized from the procedure.

\section{RESULTS AND DISCUSSION}

\section{Analytical Performance of Different Emission Lines of the Analytes}

Due to the high excitation energy of the plasma, different levels of electronic excitation take place in the analytes which leads to a large number of emission lines in the spectra. Not any emission line can be taken as an analytical line. Different merits of the analytical performance need to be investigated before choosing an emission line as the analytical lines of the elements. The most promising characteristic for an analytical line is that the response with respect to concentration should vary linearly at a sufficient length of the concentration range which is a prerequisite for the determination of the analytes. Table II summarizes the analytical performance of different lines of the analytes in aqueous medium. The data reported were on the basis of 10 replicate readings. The detection limit calculated as the concentration corresponding to the intensity equal to the average of blank values + three time of the standard deviation of the blank. The sensitivity was evaluated as the slope of the calibration curves established, while the linear dynamic range is the concentration range where the concentration and the reponse varies linearly. The line for each element with lowest detection limit, large linear dynamic range, high value of sensitivity, and best analytical performance is as follows: Ag 328.068 nm, Al 396.152 nm, 
TABLE II

Analytical Performance of Different Analytical Lines of Common Metallic Constituents and REEs

\begin{tabular}{|c|c|c|c|c|c|c|c|c|c|}
\hline & $\begin{array}{l}\text { Analytical } \\
\text { Line }\end{array}$ & $\begin{array}{l}\text { Linear Dynamic } \\
\text { Range }(\mathrm{mg} / \mathrm{L})\end{array}$ & $\begin{array}{c}\mathrm{DL} \\
(\mathrm{mg} / \mathrm{L})\end{array}$ & Sensitivity & & $\begin{array}{l}\text { Analytical } \\
\text { Line }\end{array}$ & $\begin{array}{l}\text { Linear Dynamic } \\
\text { Range }(\mathrm{mg} / \mathrm{L})\end{array}$ & $\begin{array}{c}\mathrm{DL} \\
(\mathrm{mg} / \mathrm{L})\end{array}$ & Sensitivity \\
\hline \multirow[t]{4}{*}{$\mathrm{Ag}$} & 224.641 & $4.77-1200$ & 4.76 & 132.1 & \multirow[t]{4}{*}{ Ga } & 141.444 & $12.3-1200$ & 12.3 & 8.3 \\
\hline & 243.779 & $0.162-1200$ & 0.162 & 4727.6 & & 287.424 & $6.19-1200$ & 6.19 & 107.6 \\
\hline & 328.068 & $0.022-1200$ & 0.022 & 13295.1 & & 294.364 & $1.25-1200$ & 1.25 & 861.2 \\
\hline & 338.289 & $0.0367-1200$ & 0.0367 & 8306.8 & & 417.206 & $0.015-1200$ & 0.015 & 20707.4 \\
\hline \multirow[t]{6}{*}{$\mathrm{Al}$} & 167.078 & 4.39- 1200 & 4.39 & 16.2 & \multirow[t]{4}{*}{ Gd } & 335.047 & $0.0235-2400$ & 0.0235 & 28915.8 \\
\hline & 176.641 & $4.57-1200$ & 4.57 & 8.8 & & 335.862 & $0.0387-2400$ & 0.0387 & 10967.7 \\
\hline & 308.215 & $0.237-1200$ & 0.237 & 1156.1 & & 336.223 & $0.0178-2400$ & 0.0178 & 17967.6 \\
\hline & 309.271 & $0.0329-1200$ & 0.0329 & 8102.8 & & 342.247 & $0.0116-2400$ & 0.0116 & 231975.4 \\
\hline & 394.401 & $0.017-1200$ & 0.017 & 20100.2 & \multirow[t]{4}{*}{ In } & 230.606 & $1.73-1200$ & 1.73 & 375.5 \\
\hline & 396.152 & $0.0104-1200$ & 0.0104 & 37055.2 & & 303.936 & $2.47-1200$ & 2.47 & 500.4 \\
\hline \multirow[t]{5}{*}{$\mathrm{B}$} & 136.246 & $9.25-1200$ & 9.25 & 9.7 & & 325.609 & $1.53-1200$ & 1.53 & 896.7 \\
\hline & 182.641 & $0.294-1200$ & 0.294 & 1295 & & 451.131 & $0.409-1200$ & 0.409 & 497.8 \\
\hline & 208.959 & $0.141-1200$ & 0.141 & 1784.8 & \multirow[t]{2}{*}{ K } & 404.721 & $1.51-1200$ & 1.51 & 167.4 \\
\hline & 249.667 & $0.476-1200$ & 0.476 & 1151 & & 766.461 & $0.149-1200$ & 0.149 & 1191.4 \\
\hline & 249.773 & $0.238-1200$ & 0.238 & 2415.3 & \multirow[t]{3}{*}{$\mathrm{La}$} & 333.749 & $0.0106-2400$ & 0.0106 & 82356.8 \\
\hline \multirow[t]{3}{*}{$\mathrm{Ba}$} & 230.424 & $0.157-1200$ & 0.157 & 4959.7 & & 379.478 & $0.0056-2400$ & 0.00084 & 549601.4 \\
\hline & 233.527 & $0.167-1200$ & 0.167 & 3849.1 & & 408.672 & $0.0106-2400$ & 0.000329 & 436094.1 \\
\hline & 455.404 & $0.018-1200$ & 0.018 & 105554.5 & \multirow[t]{4}{*}{$\mathrm{Li}$} & 274.118 & $1.5-1200$ & 15 & 37.3 \\
\hline \multirow[t]{7}{*}{$\mathrm{Bi}$} & 143.683 & 5.24- 1200 & 5.24 & 9.1 & & 323.261 & $3.79-1200$ & 3.79 & 206.2 \\
\hline & 153.317 & $8.97-1200$ & 8.97 & 8.6 & & 460.289 & $1.85-1200$ & 1.85 & 540.5 \\
\hline & 190.241 & $4.67-1200$ & 4.67 & 84.1 & & 670.780 & $0.00214-1200$ & 0.00214 & 135567.3 \\
\hline & 206.17 & $0.0281-1200$ & 0.0281 & 41803.2 & \multirow[t]{4}{*}{$\mathrm{Lu}$} & 219.554 & $2.58-2400$ & 2.58 & 380.4 \\
\hline & 222.825 & $4.58-1200$ & 4.58 & 115.7 & & 261.542 & $1.49-2400$ & 1.49 & 977.2 \\
\hline & 223.061 & $3.89-1200$ & 3.89 & 140.3 & & 291.139 & $2.13-2400$ & 2.13 & 711.8 \\
\hline & 306.772 & $2.71-1200$ & 2.71 & 423.6 & & 307.76 & $0.259-2400$ & 0.259 & 2667.8 \\
\hline \multirow[t]{4}{*}{$\mathrm{Ca}$} & 183.801 & $1.61-1200$ & 1.61 & 101.8 & \multirow[t]{4}{*}{$\mathrm{Mg}$} & 202.647 & $0.651-1200$ & 0.651 & 496.2 \\
\hline & 393.366 & $0.0187-1200$ & 0.0187 & 12376292 & & 279.079 & $2.42-1200$ & 2.42 & 214.9 \\
\hline & 396.847 & $0.0179-1200$ & 0.0179 & 5947582 & & 279.553 & $0.00754-1200$ & 0.00754 & 65145.1 \\
\hline & 422.673 & $0.0184-1200$ & 0.0184 & 65217.8 & & 280.27 & $0.00738-1200$ & 0.00738 & 42245.9 \\
\hline \multirow[t]{4}{*}{$\mathrm{Cd}$} & 214.438 & $0.0551-1200$ & 0.0551 & 7356.4 & & 285.213 & $0.156-1200$ & 0.156 & 5103.2 \\
\hline & 226.502 & $0.0846-1200$ & 0.0846 & 4707.8 & \multirow[t]{5}{*}{ Mn } & 257.611 & $0.0555-1200$ & 0.0555 & 14383.2 \\
\hline & 228.802 & $0.239-1200$ & 0.239 & 3330.4 & & 259.373 & $0.0672-1200$ & 0.0672 & 11850.7 \\
\hline & 361.051 & $0.0682-1200$ & 0.0682 & 2712.7 & & 260.569 & $0.0844-1200$ & 0.0844 & 8907.7 \\
\hline \multirow[t]{5}{*}{$\mathrm{Ce}$} & 393.373 & $0.364-2400$ & 0.364 & 1084913 & & 294.921 & $0.133-1200$ & 0.133 & 9945.5 \\
\hline & 413.38 & $0.0023-2400$ & 0.0023 & 95132.2 & & 403.076 & $0.0613-1200$ & 0.0613 & 11988.8 \\
\hline & 413.765 & $0.0079-2400$ & 0.0079 & 86539.8 & \multirow[t]{3}{*}{$\mathrm{Na}$} & 330.237 & $0.0277-1200$ & 0.0277 & 1761.4 \\
\hline & 418.66 & $0.0042-2400$ & 0.0042 & 95637.4 & & 330.298 & $1.3-1200$ & 1.3 & 239.5 \\
\hline & 448.691 & $0.116-2400$ & 0.116 & 5431.6 & & 588.995 & $0.0381-1200$ & 0.0381 & 55535.3 \\
\hline
\end{tabular}

Table II cont'd. 


\section{A Amic}

TABLE II (cont'd)

Analytical Performance of Different Analytical Lines of Common Metallic Constituents and REEs

\begin{tabular}{|c|c|c|c|c|c|c|c|c|c|}
\hline & $\begin{array}{l}\text { Analytical } \\
\text { Line }\end{array}$ & $\begin{array}{l}\text { Linear Dynamic } \\
\text { Range (mg/L) }\end{array}$ & $\begin{array}{c}\mathrm{DL} \\
(\mathrm{mg} / \mathrm{L})\end{array}$ & Sensitivity & & $\begin{array}{l}\text { Analytical } \\
\text { Line }\end{array}$ & $\begin{array}{l}\text { Linear Dynamic } \\
\text { Range }(\mathrm{mg} / \mathrm{L})\end{array}$ & $\begin{array}{c}\mathrm{DL} \\
(\mathrm{mg} / \mathrm{L})\end{array}$ & Sensitivity \\
\hline \multirow[t]{4}{*}{$\mathrm{Co}$} & 228.616 & $0.326-1200$ & 0.326 & 2587.6 & & & & & \\
\hline & 230.786 & $0.348-1200$ & 0.348 & 2123.1 & $\mathrm{Nd}$ & 401.225 & $0.0265-2400$ & 0.0265 & 110796 \\
\hline & 237.862 & $0.727-1200$ & 0.727 & 909.9 & & 406.109 & $0.00466-2400$ & 0.00466 & 108292.9 \\
\hline & 238.892 & $0.494-1200$ & 0.494 & 1468.4 & & 417.731 & $0.0147-2400$ & 0.0147 & 48009.1 \\
\hline \multirow[t]{5}{*}{$\mathrm{Cr}$} & 205.618 & $0.024-1200$ & 0.024 & 18014.5 & & 430.358 & $0.00821-2400$ & 0.00821 & 112295.8 \\
\hline & 267.716 & $0.246-1200$ & 0.246 & 1789.9 & $\mathrm{Ni}$ & 174.828 & $6.72-1200$ & 6.72 & 19.3 \\
\hline & 283.563 & $0.197-1200$ & 0.197 & 3667.2 & & 221.648 & $1.28-1200$ & 1.28 & 1021 \\
\hline & 284.325 & $0.458-1200$ & 0.458 & 1724.2 & & 227.021 & $1.73-1200$ & 1.73 & 60.6 \\
\hline & 284.984 & $0.595-1200$ & 0.595 & 1074.6 & & 231.604 & $0.27-1200$ & 0.27 & 2035.9 \\
\hline \multirow[t]{5}{*}{$\mathrm{Cu}$} & 219.226 & $0.89-1200$ & 0.89 & 501.6 & & 232.003 & $0.807-1200$ & 0.807 & 896.3 \\
\hline & 219.958 & $0.036-1200$ & 0.036 & 13916.7 & & 300.249 & $0.84-1200$ & 0.84 & 1851 \\
\hline & 224.7 & $0.759-1200$ & 0.759 & 762.3 & & 341.476 & $0.1-1200$ & 0.1 & 14391.2 \\
\hline & 324.754 & $0.0787-1200$ & 0.0787 & 17220.1 & $\mathrm{~Pb}$ & 167.153 & $4.97-1200$ & 4.97 & 17.6 \\
\hline & 327.396 & $0.0255-1200$ & 0.0255 & 14780.8 & & 168.215 & $6.92-1200$ & 6.92 & 17.4 \\
\hline \multirow[t]{5}{*}{ Dy } & 340.78 & $0.00164-2400$ & 0.00164 & 121163.9 & & 172.68 & $6.05-1200$ & 6.05 & 16.9 \\
\hline & 353.17 & $0.00135-2400$ & 0.00135 & 548458.6 & & 220.353 & $12.6-1200$ & 12.6 & 31.7 \\
\hline & 353.602 & $0.0029-2400$ & 0.0029 & 149494.8 & & 261.418 & $0.285-1200$ & 0.285 & 2080.5 \\
\hline & 364.54 & $0.0026-2400$ & 0.0026 & 220814.9 & & 283.305 & $8.15-1200$ & 8.15 & 87.4 \\
\hline & 394.468 & $0.00529-2400$ & 0.00529 & 176839.9 & & 405.778 & $0.222-1200$ & 0.222 & 2362.1 \\
\hline \multirow[t]{4}{*}{$\mathrm{Eu}$} & 381.967 & $0.000702-2400$ & 0.000702 & 1606502 & $\operatorname{Pr}$ & 411.846 & $0.0033-2400$ & 0.0033 & 312419.6 \\
\hline & 390.71 & $0.000871-2400$ & 0.000871 & 675848.3 & & 414.311 & $0.000994-2400$ & 0.000994 & 4311280 \\
\hline & 393.048 & $0.000739-2400$ & 0.000739 & 869738.7 & & 417.939 & $0.00147-2400$ & 0.00147 & 412745.4 \\
\hline & 420.505 & $0.000575-2400$ & 0.000575 & 1114643 & & 422.535 & $0.00102-2400$ & 0.00102 & 514045.8 \\
\hline \multirow[t]{14}{*}{$\mathrm{Fe}$} & 238.204 & $0.451-1200$ & 0.451 & 2114.9 & Sm & 359.26 & $0.00112-2400$ & 0.00112 & 119619.4 \\
\hline & 239.562 & $0.423-1200$ & 0.423 & 1906.5 & & 360.428 & $0.00129-2400$ & 0.00129 & 44879.5 \\
\hline & 241.331 & $2.86-1200$ & 2.86 & 250.9 & & 428.079 & $0.00797-2400$ & 0.00797 & 97725.2 \\
\hline & 244.451 & $10-20000$ & 10 & 59.4 & & 442.434 & $0.0123-2400$ & 0.0123 & 99263.8 \\
\hline & 259.941 & $0.388-1200$ & 0.388 & 2568.6 & $\mathrm{Sr}$ & 407.771 & $0.000506-1200$ & 0.000506 & 6548966 \\
\hline & 261.187 & $0.779-1200$ & 0.779 & 992.6 & & 421.552 & $0.000573-1200$ & 0.000573 & 3885036 \\
\hline & 262.567 & $2.34-1200$ & 2.34 & 292.9 & & 460.733 & $0.0966-1200$ & 0.0966 & 10825.2 \\
\hline & 262.829 & $2.54-1200$ & 2.54 & 277.8 & $\mathrm{Tl}$ & 132.171 & $11.5-1200$ & 11.5 & 8 \\
\hline & 275.573 & $1.39-1200$ & 1.39 & 329.1 & & 190.864 & $0.793-1200$ & 0.793 & 258.1 \\
\hline & 373.486 & $0.0118-1200$ & 0.0118 & 7034.4 & & 276.787 & $7.08-1200$ & 7.08 & 67.2 \\
\hline & 589.592 & $0.0482-1200$ & 0.0482 & 26193.5 & $\mathrm{Zn}$ & 202.613 & $0.0733-1200$ & 0.0733 & 5463.2 \\
\hline & & & & & & 206.2 & $0.144-1200$ & 0.144 & 2080.4 \\
\hline & & & & & & 213.856 & $0.0489-1200$ & 0.0489 & 5959.8 \\
\hline & & & & & & 334.502 & $0.471-1200$ & 0.471 & 745.9 \\
\hline
\end{tabular}


B $249.773 \mathrm{~nm}, \mathrm{Ba} 455.404 \mathrm{~nm}$, Bi $206.170 \mathrm{~nm}, \mathrm{Cd} 214.438 \mathrm{~nm}$, Fe $373.486 \mathrm{~nm}$, Nd $406.109 \mathrm{~nm}$, Ni $341.476 \mathrm{~nm}, \mathrm{~Pb} 405.778 \mathrm{~nm}$, K $766.461 \mathrm{~nm}, \mathrm{Li} 670.780 \mathrm{~nm}$, Dy $353.170 \mathrm{~nm}, \mathrm{Sm} 359.260 \mathrm{~nm}$, Lu $307.760 \mathrm{~nm}, \mathrm{Mg} 279.553 \mathrm{~nm}$, Sr $407.771 \mathrm{~nm}, M n 257.611 \mathrm{~nm}$, T1 190.864 nm, Zn 213.856 nm, Co 228.616 nm, Cr 205.618 nm, Cu 327.396 nm, Pr $414.311 \mathrm{~nm}$, Gd 342.247 nm, and Eu 381.967.

The Ca 396.847-nm line showed the lowest detection limit, while the 393.366-nm line was the most sensitive. Comparing both of these lines, Ca $393.366 \mathrm{~nm}$ was found to be the best with a detection limit of $0.0187 \mathrm{mg} \mathrm{L}^{-1}$ and sensitivity of 12376292. The Ce $413.380 \mathrm{~nm}$, $413.765 \mathrm{~nm}$, and $418.660 \mathrm{~nm}$ lines were found to have very good analytical performance, but the $413.380 \mathrm{~nm}$ line was found to be the best. The La 408.672-nm line showed lowest detection limits, while the La 379.478-nm line is the most sensitive. Any of these two lines can be chosen for its determination. Though the Na 330.237-nm line showed lower detection limit than $\mathrm{Na} 588.995 \mathrm{~nm}$, the latter is more than 50 times more sensitive and hence, was chosen for it analysis.

\section{Spectral Interference}

Although the presence of the additional emission lines in ICP enhances the option of choosing additional analytical lines for the determination of analytes, yet it causes severe spectral interferences. Mainly, the multi-electronic elements such as $\mathrm{Zr}$ and $\mathrm{Nb}$ are expected to interfere during the determination of analytes, especially at trace level. Hence, it leads to erroneous results of the trace metal assay. There are basically two methodolgies that can solve this issue. First, the emissionrich matrices can be preferentially removed without loss of analyte even at trace level or, second, per- form a systematic study of spectral interference of $\mathrm{Zr}$ and $\mathrm{Nb}$ and apply the correction factors where necessary. The first option requires a selective extractant and more sample handling, while the second option may not be sufficient for very low level determinations.

\section{Interference From $\mathrm{Zr}$}

The interference study includes identification of the interferencefree analytical line for the analytes, evaluation of the tolerance level of the emission-rich matrix, and determination of the correction factor for each line. Table III summarizes the parameters on the spectral interference of $\mathrm{Zr}$ on different analytes:. $\mathrm{Na} 589.529 \mathrm{~nm}$, Na $588.995 \mathrm{~nm}$, Mg $202.647 \mathrm{~nm}, \mathrm{~K} 766.491 \mathrm{~nm}$, Ca $396.847 \mathrm{~nm}$, Ca $183.801 \mathrm{~nm}$, Bi $206.170 \mathrm{~nm}$, Ce $413.765 \mathrm{~nm}$, B $182.641 \mathrm{~nm}$, B $208.959 \mathrm{~nm}$, Ni $221.648 \mathrm{~nm}, \mathrm{Zn} 213.856 \mathrm{~nm}$, and $\operatorname{Pr} 411.846 \mathrm{~nm}$ lines were found to have no spectral interference from $\mathrm{Zr}$. The $\mathrm{Cr} 205.618 \mathrm{~nm}$, Mn 259.373 nm, Fe 238.204 nm, Tl $190.864 \mathrm{~nm}$, Al $394.401 \mathrm{~nm}$, Sr $460.733 \mathrm{~nm}$, Ba $233.527 \mathrm{~nm}$, Cu $324.754 \mathrm{~nm}$, Li $670.780 \mathrm{~nm}$, Co $228.616 \mathrm{~nm}$, Ag $243.779 \mathrm{~nm}$, Lu $307.760 \mathrm{~nm}$, Ga $417.206 \mathrm{~nm}$, La $408.672 \mathrm{~nm}, \mathrm{Nd} 417.731 \mathrm{~nm}$, and $\mathrm{Eu} 390.710 \mathrm{~nm}$ analytical lines were found to be least interfered by $\mathrm{Zr}$ matrix with the tolerance level $500 \mathrm{mg} \mathrm{L}^{-1}, 100 \mathrm{mg} \mathrm{L}^{-1}, 100 \mathrm{mg} \mathrm{L}^{-1}$, $500 \mathrm{mg} \mathrm{L}^{-1}, 100 \mathrm{mg} \mathrm{L}^{-1}, 500 \mathrm{mg} \mathrm{L}^{-1}$, $100 \mathrm{mg} \mathrm{L}^{-1}, 100 \mathrm{mg} \mathrm{L}^{-1}, 100 \mathrm{mg} \mathrm{L}^{-1}$, $500 \mathrm{mg} \mathrm{L}^{-1}, 100 \mathrm{mg} \mathrm{L}^{-1}, 100 \mathrm{mg} \mathrm{L}^{-1}$, $500 \mathrm{mg} \mathrm{L}^{-1}, 500 \mathrm{mg} \mathrm{L}^{-1}, 500 \mathrm{mg} \mathrm{L}^{-1}$ and $500 \mathrm{mg} \mathrm{L}^{-1}$, respectively. Analytes like $\mathrm{Pb}$ (405.778 nm, 261.418 $\mathrm{nm}), \mathrm{Cd}(214.438 \mathrm{~nm}, 226.502$ $\mathrm{nm})$, In (230.606 nm, $451.131 \mathrm{~nm})$, Sm (359.260 nm, $442.434 \mathrm{~nm})$ and Dy (353.170 nm, $364.540 \mathrm{~nm})$ showed more than one line having least interference from $\mathrm{Zr}$ with the tolerance level of $100 \mathrm{mg} \mathrm{L}^{-1}$.

\section{Interference from $\mathbf{~ N b}$}

Since only $10-20 \%$ of $\mathrm{Nb}$ is present in the $\mathrm{Zr}-\mathrm{Nb}$ alloy, the spectral interference of $\mathrm{Nb}$ will not be as prominent as Zr. A systematic study of the spectral interference of $\mathrm{Nb}$ on other analytical wavelengths of the elements was carried out (see Table IV). The following had no interference from $\mathrm{Nb}$ : $\mathrm{Na}$ (589.592 and $588.995 \mathrm{~nm}), \mathrm{K}$ (766.491 and $404.721 \mathrm{~nm}), \mathrm{Ca}(393.366 \mathrm{~nm}$ and $183.801 \mathrm{~nm}), \operatorname{Cr}(205.618 \mathrm{~nm})$, Fe (262.567 $\mathrm{nm}$ and $373.486 \mathrm{~nm})$, $\mathrm{B}(249.773 \mathrm{~nm}$ and $182.641 \mathrm{~nm})$, $\mathrm{Al}(176.641 \mathrm{~nm}), \mathrm{Tl}(190.864 \mathrm{~nm})$, Sr (421.552 $\mathrm{nm}$ and $460.733 \mathrm{~nm})$, Bi (190.241 nm, $222.825 \mathrm{~nm}$, and $206.170 \mathrm{~nm}), \mathrm{Li}(670.780 \mathrm{~nm})$, Co (228.616 nm, $230.786 \mathrm{~nm}$, and $237.862 \mathrm{~nm}), \mathrm{Ni}(231.604 \mathrm{~nm}$, $221.648 \mathrm{~nm}$, and $341.476 \mathrm{~nm}$ ), $\mathrm{Zn}(213.856 \mathrm{~nm}$ and $206.200 \mathrm{~nm})$, $\mathrm{Ag}(328.068 \mathrm{~nm}), \mathrm{Cd}(361.051 \mathrm{~nm})$, $\mathrm{Ga}(417.206 \mathrm{~nm})$, In (230.606 nm, $451.131 \mathrm{~nm}, 460.289 \mathrm{~nm}$, and $323.261 \mathrm{~nm}), \mathrm{La}(408.672 \mathrm{~nm}$, $333.749 \mathrm{~nm}$, and $374.478 \mathrm{~nm}$ ), Ce $(418.660 \mathrm{~nm}, 448.691 \mathrm{~nm}$, $413.380 \mathrm{~nm}, 393.373 \mathrm{~nm})$, $\operatorname{Pr}(411.846 \mathrm{~nm}, 417.939 \mathrm{~nm}$, and $422.535 \mathrm{~nm}), \mathrm{Nd}(430.358 \mathrm{~nm}$, $417.731 \mathrm{~nm}), \mathrm{Sm}(359.260 \mathrm{~nm}$, $442.434 \mathrm{~nm}, 428.079 \mathrm{~nm}$, and $360.428 \mathrm{~nm})$, Eu (381.967 nm, $393.048 \mathrm{~nm}, 390.710 \mathrm{~nm}$ ) and Dy (353.170 nm, 394.468 nm). For other analytes, the least interferred lines were identified along with their tolerance level and correction factors. The analaytical lines of $\mathrm{Mn}$ $260.569 \mathrm{~nm}, \mathrm{~Pb} 405.778 \mathrm{~nm}, \mathrm{Cu}$ $327.396 \mathrm{~nm}, \mathrm{Gd} 335.047 \mathrm{~nm}$, and Lu $361.542 \mathrm{~nm}$ were found to show the least interference from $\mathrm{Nb}$ with a tolerance level of $500 \mathrm{mg} \mathrm{L}^{-1}$ for all except $\mathrm{Cu}$ at $327.396 \mathrm{~nm}$ (tolerance of $\mathrm{Nb} 200 \mathrm{mg} \mathrm{L}^{-1}$ ).

The overall study revealed that, comparatively, the spectral interference of $\mathrm{Zr}$ showed a more pronounced effect during the analysis of the trace metals. Therefore, it is a prerequisite to separate $\mathrm{Zr}$ preferentially without any loss of the analytes even at the trace level. 


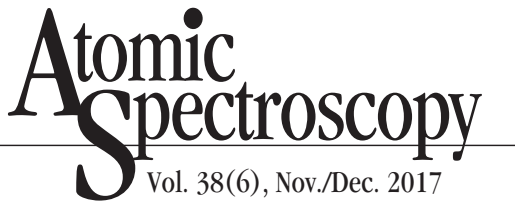

TABLE III

Spectral Interference of $\mathrm{Zr}$ on Different Analytical Lines of the Elements

\begin{tabular}{|c|c|c|c|c|c|c|c|c|c|}
\hline & $\begin{array}{l}\text { Analytical } \\
\text { Line } \\
\text { (nm) }\end{array}$ & $\begin{array}{l}\text { Contri- } \\
\text { bution } \\
\text { From } \\
1000 \\
\text { ppm Zr }\end{array}$ & $\begin{array}{l}\text { Toler- } \\
\text { ance } \\
\text { Level } \\
(\mathrm{mg} / \mathrm{L})\end{array}$ & Correction Factor & & $\begin{array}{l}\text { Analytical } \\
\text { Line } \\
\text { (nm) }\end{array}$ & $\begin{array}{l}\text { Contri- } \\
\text { bution } \\
\text { From } \\
1000 \\
\text { ppm Zr }\end{array}$ & $\begin{array}{l}\text { Toler- } \\
\text { ance } \\
\text { Level } \\
(\mathrm{mg} / \mathrm{L})\end{array}$ & Correction Factor \\
\hline \multirow[t]{4}{*}{$\mathrm{Na}$} & 589.592 & $\mathrm{BDL}$ & 1000 & NO INTERFERENCE & B & 249.773 & 7.605 & 0.5 & 0.007605 \\
\hline & 588.995 & BDL & 1000 & NO INTERFERENCE & & 249.677 & 22.222 & 1 & 0.022222 \\
\hline & 330.237 & 96.512 & 0.05 & 0.096512 & & 182.641 & BDL & 1000 & NO INTERFERENCE \\
\hline & 330.298 & 436.878 & 20 & 0.436878 & & 208.959 & BDL & 1000 & NO INTERFERENCE \\
\hline \multirow[t]{5}{*}{$\mathrm{Mg}$} & 279.553 & 0.462 & 0.05 & 0.000462 & & 136.246 & 394.428 & 20 & 0.394428 \\
\hline & 280.27 & 0.459 & 0.05 & 0.000459 & $\mathrm{Al}$ & 167.078 & 361.303 & 20 & 0.361303 \\
\hline & 285.213 & 71.633 & 0.05 & 0.071633 & & 396.152 & 57.081 & 1 & 0.057081 \\
\hline & 279.079 & 85.762 & 100 & 0.085762 & & 394.401 & 1.29 & 100 & 0.00129 \\
\hline & 202.647 & BDL & 1000 & NO INTERFERENCE & & 176.641 & 325.454 & 50 & 0.325454 \\
\hline \multirow[t]{2}{*}{ K } & 766.491 & $\mathrm{BDL}$ & 1000 & NO INTERFERENCE & & 308.215 & 17.019 & 100 & 0.017019 \\
\hline & 404.721 & 551.068 & 20 & 0.551068 & & 309.271 & 6.273 & 1 & 0.006273 \\
\hline \multirow[t]{4}{*}{$\mathrm{Ca}$} & 396.847 & $\mathrm{BDL}$ & 1000 & NO INTERFERENCE & $\mathrm{Tl}$ & 190.864 & 3.566 & 500 & 0.003566 \\
\hline & 393.366 & 0.039 & 500 & 0.000039 & & 132.171 & 459.833 & 20 & 0.459833 \\
\hline & 183.801 & $\mathrm{BDL}$ & 1000 & NO INTERFERENCE & & 276.787 & 253.539 & 50 & 0.253539 \\
\hline & 422.673 & 0.826 & 10 & 0.000826 & $\mathrm{Sr}$ & 407.771 & 0.003 & 100 & 0.000003 \\
\hline \multirow[t]{5}{*}{$\mathrm{Cr}$} & 267.716 & 14.87 & 100 & 0.01487 & & 421.552 & 0.039 & 100 & 0.000039 \\
\hline & 205.618 & 0.068 & 500 & 0.000068 & & 460.733 & 0.135 & 500 & 0.000135 \\
\hline & 283.563 & 97.01 & 1 & 0.09701 & $\mathrm{Ba}$ & 455.404 & 0.538 & 50 & 0.000538 \\
\hline & 284.325 & 81.506 & 0.05 & 0.081506 & & 233.527 & 1.221 & 100 & 0.001221 \\
\hline & 284.984 & 39.206 & 100 & 0.039206 & & 230.424 & 3.02 & 100 & 0.00302 \\
\hline \multirow[t]{5}{*}{$\mathrm{Mn}$} & 257.611 & 3.998 & 20 & 0.003998 & $\mathrm{Bi}$ & 223.061 & 21.43 & 500 & 0.02143 \\
\hline & 259.373 & 1.577 & 100 & 0.001577 & & 190.241 & 136.809 & 100 & 0.136809 \\
\hline & 260.569 & 4.912 & 100 & 0.004912 & & 306.772 & 158.407 & 0.05 & 0.158407 \\
\hline & 294.921 & 26.509 & 10 & 0.026509 & & 222.825 & 8.139 & 500 & 0.008139 \\
\hline & 403.076 & 10.351 & 20 & 0.010351 & & 153.317 & 404.799 & 50 & 0.404799 \\
\hline \multirow[t]{4}{*}{$\mathrm{Fe}$} & 259.941 & 4.448 & 10 & 0.004448 & & 143.683 & 388.723 & 20 & 0.388723 \\
\hline & 238.204 & 5.264 & 100 & 0.005264 & & 206.17 & $\mathrm{BDL}$ & 1000 & NO INTERFERENCE \\
\hline & 239.562 & 4.81 & 10 & 0.00481 & $\mathrm{~Pb}$ & 220.353 & 112.962 & 100 & 0.112962 \\
\hline & 244.451 & 385.825 & 100 & 0.385825 & & 168.215 & 345.695 & 20 & 0.345695 \\
\hline
\end{tabular}

TABLE III (cont'd.) 
TABLE III (cont'd.)

Spectral Interference of $\mathrm{Zr}$ on Different Analytical Lines of The Elements

\begin{tabular}{|c|c|c|c|c|c|c|c|c|c|}
\hline & $\begin{array}{l}\text { Analytical } \\
\text { Line } \\
\text { (nm) }\end{array}$ & $\begin{array}{l}\text { Contri- } \\
\text { bution } \\
\text { From } \\
1000 \\
\text { ppm Zr }\end{array}$ & $\begin{array}{l}\text { Toler- } \\
\text { ance } \\
\text { Level } \\
(\mathrm{mg} / \mathrm{L})\end{array}$ & Correction Factor & & $\begin{array}{l}\text { Analytical } \\
\text { Line } \\
\text { (nm) }\end{array}$ & $\begin{array}{l}\text { Contri- } \\
\text { bution } \\
\text { From } \\
1000 \\
\text { ppm Zr }\end{array}$ & $\begin{array}{l}\text { Toler- } \\
\text { ance } \\
\text { Level } \\
(\mathrm{mg} / \mathrm{L})\end{array}$ & Correction Factor \\
\hline \multirow[t]{6}{*}{$\mathrm{Fe}$} & 261.187 & 15.177 & 10 & 0.015177 & \multirow[t]{5}{*}{$\mathrm{Pb}$} & 167.153 & 335.923 & 20 & 0.335923 \\
\hline & 262.567 & 26.534 & 100 & 0.026534 & & 172.68 & 359.134 & 20 & 0.359134 \\
\hline & 262.829 & 41.608 & 100 & 0.041608 & & 405.778 & 3.321 & 100 & 0.003321 \\
\hline & 275.573 & 36.526 & 50 & 0.036526 & & 283.305 & 148.822 & 100 & 0.148822 \\
\hline & 241.331 & 31.081 & 100 & 0.031081 & & 261.418 & 3.768 & 100 & 0.003768 \\
\hline & 373.486 & 3.301 & 100 & 0.003301 & \multirow[t]{4}{*}{$\mathrm{Li}$} & 670.78 & 0.019 & 100 & 0.000019 \\
\hline \multirow[t]{4}{*}{ Co } & 228.616 & 1.739 & 500 & 0.001739 & & 460.289 & 76.117 & 100 & 0.076117 \\
\hline & 238.892 & 10.867 & 100 & 0.010867 & & 323.261 & 214.029 & 20 & 0.214029 \\
\hline & 230.786 & 2.856 & 100 & 0.002856 & & 274.118 & 2161.41 & 10 & 2.16141 \\
\hline & 237.862 & 84.807 & 20 & 0.084807 & \multirow[t]{3}{*}{$\mathrm{La}$} & 408.672 & 0.014 & 500 & 0.000014 \\
\hline \multirow[t]{7}{*}{$\mathrm{Ni}$} & 231.604 & 5.689 & 100 & 0.005689 & & 333.749 & 3.238 & 20 & 0.003238 \\
\hline & 221.648 & $\mathrm{BDL}$ & 1000 & NO INTERFERENCE & & 379.478 & 0.047 & 100 & 0.000047 \\
\hline & 232.003 & 8.714 & 100 & 0.008714 & \multirow[t]{5}{*}{$\mathrm{Ce}$} & 418.66 & 256.962 & 1 & 0.256962 \\
\hline & 227.021 & 38.182 & 100 & 0.038182 & & 448.691 & 6.831 & 100 & 0.006831 \\
\hline & 174.828 & 374.178 & 20 & 0.374178 & & 413.765 & $\mathrm{BDL}$ & 1000 & NO INTERFERENCE \\
\hline & 300.249 & 12.387 & 100 & 0.012387 & & 413.38 & 1.627 & 100 & 0.001627 \\
\hline & 341.476 & 174.381 & 0.1 & 0.174381 & & 393.373 & 1.177 & 100 & 0.001177 \\
\hline \multirow[t]{5}{*}{$\mathrm{Cu}$} & 324.754 & 0.962 & 100 & 0.000962 & \multirow[t]{4}{*}{$\operatorname{Pr}$} & 411.846 & $\mathrm{BDL}$ & 1000 & NO INTERFERENCE \\
\hline & 327.396 & 23.038 & 5 & 0.023038 & & 417.939 & 1.031 & 100 & 0.001031 \\
\hline & 224.7 & 7.952 & 100 & 0.007952 & & 422.535 & 0.584 & 20 & 0.000584 \\
\hline & 219.226 & 8.732 & 100 & 0.008732 & & 414.311 & 0.218 & 100 & 0.000218 \\
\hline & 219.958 & 1.738 & 100 & 0.001738 & \multirow[t]{4}{*}{$\mathrm{Nd}$} & 401.225 & 10.57 & 10 & 0.01057 \\
\hline \multirow[t]{4}{*}{$\mathrm{Zn}$} & 213.856 & $\mathrm{BDL}$ & 1000 & NO INTERFERENCE & & 430.358 & 0.253 & 100 & 0.000253 \\
\hline & 206.2 & 0.578 & 500 & 0.000578 & & 406.109 & 0.323 & 100 & 0.000323 \\
\hline & 202.613 & 0.454 & 100 & 0.000454 & & 417.731 & 0.188 & 500 & 0.000188 \\
\hline & 334.502 & 704.9 & 1 & 0.7049 & \multirow[t]{4}{*}{$\mathrm{Sm}$} & 359.26 & 0.197 & 100 & 0.000197 \\
\hline \multirow[t]{4}{*}{$\mathrm{Ag}$} & 328.068 & 414.093 & 0.5 & 0.414093 & & 442.434 & 0.148 & 100 & 0.000148 \\
\hline & 338.289 & 4.256 & 50 & 0.004256 & & 428.079 & 0.295 & 100 & 0.000295 \\
\hline & 224.641 & 61.563 & 100 & 0.061563 & & 360.428 & 0.959 & 100 & 0.000959 \\
\hline & 243.779 & 1.701 & 100 & 0.001701 & \multirow[t]{4}{*}{$\mathrm{Eu}$} & 420.505 & 0.029 & 100 & 0.000029 \\
\hline \multirow[t]{4}{*}{$\mathrm{Cd}$} & 214.438 & 1.024 & 100 & 0.001024 & & 381.967 & 0.365 & 100 & 0.000365 \\
\hline & 226.502 & 1.134 & 100 & 0.001134 & & 393.048 & 0.052 & 100 & 0.000052 \\
\hline & 228.802 & 2.09 & 100 & 0.00209 & & 390.71 & 0.004 & 500 & 0.000004 \\
\hline & 361.051 & 40.852 & 20 & 0.040852 & \multirow[t]{4}{*}{ Gd } & 342.247 & 0.276 & 100 & 0.000276 \\
\hline \multirow[t]{4}{*}{$\mathrm{Ga}$} & 141.444 & 419.055 & 50 & 0.419055 & & 335.047 & 47.552 & 1 & 0.047552 \\
\hline & 417.206 & 0.147 & 500 & 0.000147 & & 336.223 & 8.38 & 0.05 & 0.00838 \\
\hline & 294.364 & 22.834 & 100 & 0.022834 & & 335.862 & 13.839 & 20 & 0.013839 \\
\hline & 287.424 & 324.653 & 50 & 0.324653 & \multirow[t]{5}{*}{ Dy } & 353.17 & 0.073 & 100 & 0.000073 \\
\hline \multirow[t]{8}{*}{ In } & 230.606 & 14.581 & 100 & 0.014581 & & 394.468 & 0.16 & 100 & 0.00016 \\
\hline & 325.609 & 143.989 & 20 & 0.143989 & & 340.78 & 14.05 & 1 & 0.01405 \\
\hline & 451.131 & 11.426 & 100 & 0.011426 & & 353.602 & 1.79 & 20 & 0.00179 \\
\hline & 303.936 & 253.123 & 20 & 0.253123 & & 364.54 & 0.083 & 100 & 0.000083 \\
\hline & & & & & \multirow[t]{4}{*}{$\mathrm{Lu}$} & 261.542 & 59.116 & 50 & 0.059116 \\
\hline & & & & & & 291.139 & 36.806 & 100 & 0.036806 \\
\hline & & & & & & 307.76 & 8.752 & 100 & 0.008752 \\
\hline & & & & & & 219.554 & 17.99 & 100 & 0.01799 \\
\hline
\end{tabular}




\section{A Amic}

TABLE IV

Spectral Interference of $\mathrm{Nb}$ on Different Analytical Lines of the Elements

\begin{tabular}{|c|c|c|c|c|c|c|c|c|c|}
\hline & $\begin{array}{l}\text { Analytical } \\
\text { Line } \\
\text { (nm) }\end{array}$ & $\begin{array}{l}\text { Contri- } \\
\text { bution } \\
\text { From } \\
1000 \\
\text { ppm Nb }\end{array}$ & $\begin{array}{l}\text { Toler- } \\
\text { ance } \\
\text { Level } \\
(\mathrm{mg} / \mathrm{L})\end{array}$ & Correction Factor & & $\begin{array}{l}\text { Analytical } \\
\text { Line } \\
\text { (nm) }\end{array}$ & $\begin{array}{l}\text { Contri- } \\
\text { bution } \\
\text { From } \\
1000 \\
\text { ppm Nb }\end{array}$ & $\begin{array}{l}\text { Toler- } \\
\text { ance } \\
\text { Level } \\
(\mathrm{mg} / \mathrm{L})\end{array}$ & Correction Factor \\
\hline \multirow[t]{4}{*}{$\mathrm{Na}$} & 589.592 & BDL & 1000 & NO INTERFERENCE & \multirow[t]{5}{*}{$\mathrm{B}$} & 249.773 & $\mathrm{BDL}$ & 1000 & NO INTERFERENCE \\
\hline & 588.995 & $\mathrm{BDL}$ & 1000 & NO INTERFERENCE & & 249.677 & 16.782 & 100 & 0.016782 \\
\hline & 330.237 & 6.095 & 0.05 & 0.006095 & & 182.641 & $\mathrm{BDL}$ & 1000 & NO INTERFERENCE \\
\hline & 330.298 & 32.849 & 100 & 0.032849 & & 208.959 & 1.237 & 100 & 0.001237 \\
\hline \multirow[t]{5}{*}{$\mathrm{Mg}$} & 279.553 & 1.085 & 0.05 & 0.001085 & & 136.246 & 30.359 & 500 & 0.030359 \\
\hline & 280.27 & 1.652 & 0.05 & 0.001652 & \multirow[t]{6}{*}{$\mathrm{Al}$} & 167.078 & 15.692 & 500 & 0.015692 \\
\hline & 285.213 & 1.287 & 0.1 & 0.001287 & & 396.152 & 1.554 & 50 & 0.001554 \\
\hline & 279.079 & 268.903 & 20 & 0.268903 & & 394.401 & 0.077 & 500 & 0.000077 \\
\hline & 202.647 & BDL & 1000 & NO INTERFERENCE & & 176.641 & $\mathrm{BDL}$ & 1000 & NO INTERFERENCE \\
\hline \multirow[t]{2}{*}{$\mathrm{K}$} & 766.491 & BDL & 1000 & NO INTERFERENCE & & 308.215 & 4.896 & 50 & 0.004896 \\
\hline & 404.721 & BDL & 1000 & NO INTERFERENCE & & 309.271 & 6.148 & 1 & 0.006148 \\
\hline \multirow[t]{4}{*}{$\mathrm{Ca}$} & 396.847 & 0.069 & 0.1 & 0.000069 & \multirow[t]{3}{*}{$\mathrm{Tl}$} & 190.864 & $\mathrm{BDL}$ & 1000 & NO INTERFERENCE \\
\hline & 393.366 & BDL & 1000 & NO INTERFERENCE & & 132.171 & 45.262 & 100 & 0.045262 \\
\hline & 183.801 & BDL & 1000 & NO INTERFERENCE & & 276.787 & 1534.79 & 20 & 1.53479 \\
\hline & 422.673 & 0.06 & 0.1 & 0.00006 & \multirow[t]{3}{*}{$\mathrm{Sr}$} & 407.771 & 0.005 & 50 & 0.000005 \\
\hline \multirow[t]{5}{*}{$\mathrm{Cr}$} & 267.716 & 0.881 & 500 & 0.000881 & & 421.552 & $\mathrm{BDL}$ & 1000 & NO INTERFERENCE \\
\hline & 205.618 & BDL & 1000 & NO INTERFERENCE & & 460.733 & $\mathrm{BDL}$ & 1000 & NO INTERFERENCE \\
\hline & 283.563 & 0.67 & 500 & 0.00067 & \multirow[t]{3}{*}{$\mathrm{Ba}$} & 455.404 & $\mathrm{BDL}$ & 1000 & NO INTERFERENCE \\
\hline & 284.325 & 9.306 & 5 & 0.009306 & & 233.527 & 82.845 & 20 & 0.082845 \\
\hline & 284.984 & 2.203 & 500 & 0.002203 & & 230.424 & 0.214 & 500 & 0.000214 \\
\hline \multirow[t]{5}{*}{$\mathrm{Mn}$} & 257.611 & 0.284 & 500 & 0.000284 & \multirow[t]{7}{*}{$\mathrm{Bi}$} & 223.061 & 173.656 & 100 & 0.173656 \\
\hline & 259.373 & 14.964 & 20 & 0.014964 & & 190.241 & $\mathrm{BDL}$ & 1000 & NO INTERFERENCE \\
\hline & 260.569 & 0.194 & 500 & 0.000194 & & 306.772 & 36.661 & 100 & 0.036661 \\
\hline & 294.921 & 1.555 & 100 & 0.001555 & & 222.825 & $\mathrm{BDL}$ & 1000 & NO INTERFERENCE \\
\hline & 403.076 & 0.747 & 100 & 0.000747 & & 153.317 & 16.58 & 500 & 0.01658 \\
\hline \multirow[t]{10}{*}{$\mathrm{Fe}$} & 259.941 & 20.946 & 10 & 0.020946 & & 143.683 & 22.786 & 500 & 0.022786 \\
\hline & 238.204 & 3.941 & 10 & 0.003941 & & 206.17 & $\mathrm{BDL}$ & 1000 & NO INTERFERENCE \\
\hline & 239.562 & 5.078 & 10 & 0.005078 & \multirow[t]{7}{*}{$\mathrm{Pb}$} & 220.353 & 25620 & 20 & 25.62 \\
\hline & 244.451 & 3117.97 & 20 & 3.11797 & & 168.215 & 19.597 & 500 & 0.019597 \\
\hline & 261.187 & 5.43 & 20 & 0.00543 & & 167.153 & 18.251 & 500 & 0.018251 \\
\hline & 262.567 & BDL & 1000 & NO INTERFERENCE & & 172.68 & 21.598 & 500 & 0.021598 \\
\hline & 262.829 & 93.947 & 50 & 0.093947 & & 405.778 & 2.422 & 500 & 0.002422 \\
\hline & 275.573 & 22.137 & 100 & 0.022137 & & 283.305 & 9.322 & 500 & 0.009322 \\
\hline & 241.331 & 14.022 & 100 & 0.014022 & & 261.418 & 8.121 & 100 & 0.008121 \\
\hline & 373.486 & BDL & 1000 & NO INTERFERENCE & \multirow[t]{5}{*}{$\mathrm{Li}$} & 670.78 & $\mathrm{BDL}$ & 1000 & NO INTERFERENCE \\
\hline \multirow[t]{4}{*}{ Co } & 228.616 & $\mathrm{BDL}$ & 1000 & NO INTERFERENCE & & 460.289 & $\mathrm{BDL}$ & 1000 & NO INTERFERENCE \\
\hline & 238.892 & 0.762 & 500 & 0.000762 & & 323.261 & BDL & 1000 & NO INTERFERENCE \\
\hline & 230.786 & $\mathrm{BDL}$ & 1000 & NO INTERFERENCE & & 274.118 & 524.647 & 100 & 0.524647 \\
\hline & 237.862 & BDL & 1000 & NO INTERFERENCE & & & & & \\
\hline
\end{tabular}

TABLE IV (cont'd.) 
TABLE IV (cont'd.)

Spectral Interference of $\mathrm{Nb}$ on Different Analytical Lines of The Elements

\begin{tabular}{|c|c|c|c|c|c|c|c|c|c|}
\hline & $\begin{array}{l}\text { Analytical } \\
\text { Line } \\
\text { (nm) }\end{array}$ & $\begin{array}{l}\text { Contri- } \\
\text { bution } \\
\text { From } \\
1000 \\
\text { ppm Nb }\end{array}$ & $\begin{array}{l}\text { Toler- } \\
\text { ance } \\
\text { Level } \\
(\mathrm{mg} / \mathrm{L})\end{array}$ & Correction Factor & & $\begin{array}{l}\text { Analytical } \\
\text { Line } \\
\text { (nm) }\end{array}$ & $\begin{array}{l}\text { Contri- } \\
\text { bution } \\
\text { From } \\
1000 \\
\text { ppm Nb }\end{array}$ & $\begin{array}{l}\text { Toler- } \\
\text { ance } \\
\text { Level } \\
\text { (mg/L) }\end{array}$ & Correction Factor \\
\hline & & & & & $\mathrm{La}$ & 408.672 & BDL & 1000 & NO INTERFERENCE \\
\hline \multirow{7}{*}{$\mathrm{Ni}$} & 231.604 & BDL & 1000 & NO INTERFERENCE & & 333.749 & BDL & 1000 & NO INTERFERENCE \\
\hline & 221.648 & BDL & 1000 & NO INTERFERENCE & & 379.478 & BDL & 1000 & NO INTERFERENCE \\
\hline & 232.003 & 30.543 & 100 & 0.030543 & $\mathrm{Ce}$ & 418.66 & BDL & 1000 & NO INTERFERENCE \\
\hline & 227.021 & 1177.4 & 20 & 1.1774 & & 448.691 & $\mathrm{BDL}$ & 1000 & NO INTERFERENCE \\
\hline & 174.828 & 25.449 & 500 & 0.025449 & & 413.765 & 0.296 & 100 & 0.000296 \\
\hline & 300.249 & 10.263 & 100 & 0.010263 & & 413.38 & $\mathrm{BDL}$ & 1000 & NO INTERFERENCE \\
\hline & 341.476 & $\mathrm{BDL}$ & 1000 & NO INTERFERENCE & & 393.373 & BDL & 1000 & NO INTERFERENCE \\
\hline \multirow[t]{5}{*}{$\mathrm{Cu}$} & 324.754 & 67.114 & 20 & 0.067114 & $\operatorname{Pr}$ & 411.846 & BDL & 1000 & NO INTERFERENCE \\
\hline & 327.396 & 9.97 & 200 & 0.00997 & & 417.939 & BDL & 1000 & NO INTERFERENCE \\
\hline & 224.7 & 39.334 & 100 & 0.039334 & & 422.535 & BDL & 1000 & NO INTERFERENCE \\
\hline & 219.226 & 12.436 & 100 & 0.012436 & & 414.311 & 0.276 & 100 & 0.000276 \\
\hline & 219.958 & 28.782 & 20 & 0.028782 & $\mathrm{Nd}$ & 401.225 & 1.483 & 50 & 0.001483 \\
\hline \multirow[t]{4}{*}{$\mathrm{Zn}$} & 213.856 & BDL & 1000 & NO INTERFERENCE & & 430.358 & BDL & 1000 & NO INTERFERENCE \\
\hline & 206.2 & $\mathrm{BDL}$ & 1000 & NO INTERFERENCE & & 406.109 & 0.203 & 100 & 0.000203 \\
\hline & 202.613 & 6.174 & 50 & 0.006174 & & 417.731 & BDL & 1000 & NO INTERFERENCE \\
\hline & 334.502 & 24.767 & 100 & 0.024767 & $\mathrm{Sm}$ & 359.26 & BDL & 1000 & NO INTERFERENCE \\
\hline \multirow[t]{4}{*}{ Ag } & 328.068 & BDL & 1000 & NO INTERFERENCE & & 442.434 & BDL & 1000 & NO INTERFERENCE \\
\hline & 338.289 & 0.426 & 500 & 0.000426 & & 428.079 & BDL & 1000 & NO INTERFERENCE \\
\hline & 224.641 & 812.777 & 20 & 0.812777 & & 360.428 & $\mathrm{BDL}$ & 1000 & NO INTERFERENCE \\
\hline & 243.779 & 0.758 & 500 & 0.000758 & $\mathrm{Eu}$ & 420.505 & 0.018 & 100 & 0.000018 \\
\hline \multirow[t]{4}{*}{$\mathrm{Cd}$} & 214.438 & 1.041 & 100 & 0.001041 & & 381.967 & BDL & 1000 & NO INTERFERENCE \\
\hline & 226.502 & 1.731 & 500 & 0.001731 & & 393.048 & $\mathrm{BDL}$ & 1000 & NO INTERFERENCE \\
\hline & 228.802 & 0.869 & 500 & 0.000869 & & 390.71 & BDL & 1000 & NO INTERFERENCE \\
\hline & 361.051 & $\mathrm{BDL}$ & 1000 & NO INTERFERENCE & Gd & 342.247 & 0.72 & 100 & 0.00072 \\
\hline \multirow[t]{4}{*}{ Ga } & 141.444 & 18.845 & 500 & 0.018845 & & 335.047 & 0.217 & 500 & 0.000217 \\
\hline & 417.206 & BDL & 1000 & NO INTERFERENCE & & 336.223 & 5.214 & 0.1 & 0.005214 \\
\hline & 294.364 & 5.507 & 500 & 0.005507 & & 335.862 & 7.148 & 50 & 0.007148 \\
\hline & 287.424 & 44.556 & 100 & 0.044556 & Dy & 353.17 & BDL & 1000 & NO INTERFERENCE \\
\hline \multirow[t]{8}{*}{ In } & 230.606 & BDL & 1000 & NO INTERFERENCE & & 394.468 & BDL & 1000 & NO INTERFERENCE \\
\hline & 325.609 & 8.495 & 100 & 0.008495 & & 340.78 & 0.418 & 100 & 0.000418 \\
\hline & 451.131 & $\mathrm{BDL}$ & 1000 & NO INTERFERENCE & & 353.602 & 0.018 & 500 & 0.000018 \\
\hline & 303.936 & 6186.35 & 10 & 6.18635 & & 364.54 & 0.032 & 100 & 0.000032 \\
\hline & & & & & $\mathrm{Lu}$ & 261.542 & 3.333 & 500 & 0.003333 \\
\hline & & & & & & 291.139 & 157.447 & 50 & 0.157447 \\
\hline & & & & & & 307.76 & 9.801 & 100 & 0.009801 \\
\hline & & & & & & 219.554 & 110.51 & 100 & 0.11051 \\
\hline
\end{tabular}




\section{Atomic Apectroscopy \\ $\bigcup$ Vol. 38(6), Nov./Dec. 2017}

\section{Preferential Separation of $\mathbf{Z r}$ Without Loss of Analytes}

For the efficient separation of $\mathrm{Zr}$, three extractants, i.e., tri-n-butyl phosphate (TBP), di-n-hexyl octanamide (DHOA), and tri-n-octyl phosphine oxide (TOPO) were selected. A $1.1 \mathrm{M}$ concentration of each ligand was used for the preferential separation of $\mathrm{Zr}$. The extraction profiles were established at different aqueous feed acidity ranging from $0.01 \mathrm{M} \mathrm{HNO}_{3}$ to $6 \mathrm{M} \mathrm{HNO}_{3}$ (Figure 1). The $\mathrm{D}_{\mathrm{Zr}}$ value was found to increase with an increase in aqueous feed acidity up to $3 \mathrm{M}$ $\mathrm{HNO}_{3}$ with a $\mathrm{D}_{\mathrm{Zr}}$ value of 4,3 , and 1.9 for TOPO, TBP, and DHOA, respectively. This initial increase in $D$ values with an increase in aqueous feed acidity can be attributed to the participation of the nitrate ion during complexation. Beyond $3 \mathrm{M}$ $\mathrm{HNO}_{3}$, this drastic enhancement was found to be destroyed due to the competition of the $\mathrm{H}^{+}$ion with $\mathrm{Zr}^{4+}$. The trend in $\mathrm{D}_{\mathrm{Zr}}$ values using these three ligands can be explained on the basis of their electron donating ability. Based on this investigation, the aqueous feed acidity for processing of the actual $\mathrm{Zr}-\mathrm{Nb}$ samples was optimized as $3 \mathrm{M} \mathrm{HNO}_{3}$.

Since the above study revealed that all of these ligands can be used for the efficient extraction of $\mathrm{Zr}$, it also requires to know the fate of the analytes while processing with these solvent systems. Based on the analytical performance of different analytical lines of the elements and the spectral interference of $\mathrm{Zr}$ and $\mathrm{Nb}$, the lines of $\mathrm{Al} 394.401 \mathrm{~nm}$, B $182.641 \mathrm{~nm}, \mathrm{Ba} 455.404 \mathrm{~nm}$, Bi $206.170 \mathrm{~nm}$, Ca $393.366 \mathrm{~nm}$, Cd $226.502 \mathrm{~nm}$, Ce $413.760 \mathrm{~nm}$, Ga 417.206 nm, Gd 342.247 nm, In $451.131 \mathrm{~nm}, \mathrm{~K} 766.491 \mathrm{~nm}$, La $408.672 \mathrm{~nm}, \mathrm{Li} 670.780 \mathrm{~nm}$, Lu $307.760 \mathrm{~nm}, \mathrm{Mg} 279.553 \mathrm{~nm}$, Mn $257.611 \mathrm{~nm}, \mathrm{Na} 588.995 \mathrm{~nm}$, Co 228.616 nm, Cr 205.618 nm, Cu $219.958 \mathrm{~nm}$, Dy $353.170 \mathrm{~nm}$, Eu 390.710 nm, Fe 373.486 nm,

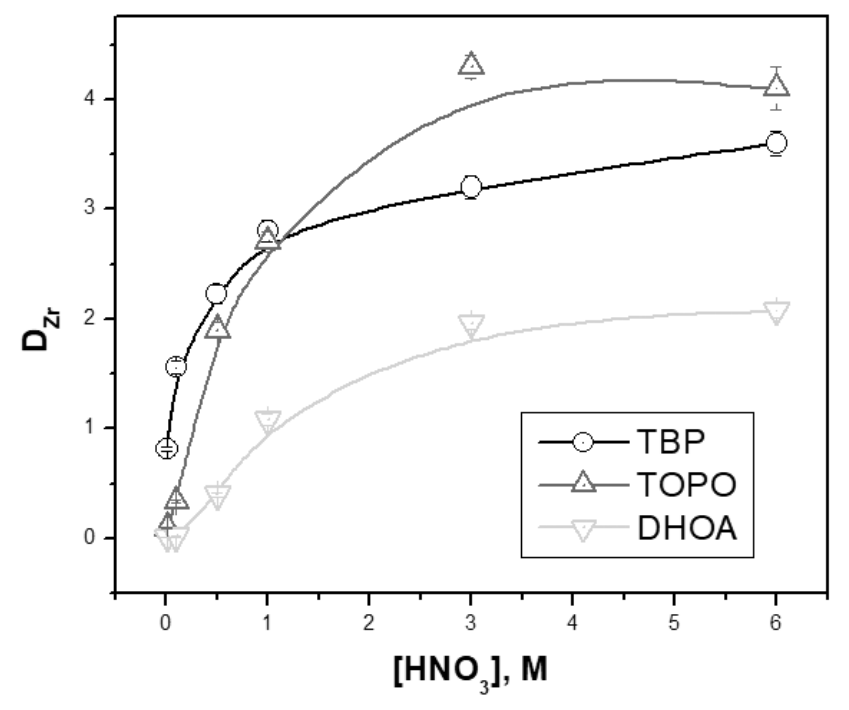

Fig. 1. The distribution ratio values for $\mathrm{Zr}$ at different aqueous feed acidity.

Nd $430.358 \mathrm{~nm}$, Ni $231.604 \mathrm{~nm}$, $\mathrm{Pb} 405.778 \mathrm{~nm}, \operatorname{Pr} 417.939 \mathrm{~nm}$, Sm $359.260 \mathrm{~nm}$, Sr $421.556 \mathrm{~nm}$, T1 $190.864 \mathrm{~nm}$, and $\mathrm{Zn} 213.856 \mathrm{~nm}$ are suitable for their determination in a $\mathrm{Zr}-\mathrm{Nb}$ matrix.

\section{Determination of Number of Contacts}

One contact of the organic phase containing a ligand in dodecane was not sufficient to bring the $\mathrm{Zr}$ level to below the tolerance level of the specified analytical lines. Starting from $10,000 \mathrm{mg} \mathrm{L}^{-1}$ concentration of $\mathrm{Zr}$, the separation process was carried out with equal volume of the organic phase. After each contact, the raffinate was analyzed for residual $\mathrm{Zr}$. In most cases the tolerance level for $\mathrm{Zr}$ and $\mathrm{Nb}$ was above $100 \mathrm{mg} \mathrm{L}^{-1}$ (except the $\mathrm{Zr}$ tolerance for $\mathrm{Ag} 338.289 \mathrm{~nm}, \mathrm{Ba}$ $455.404 \mathrm{~nm}, \mathrm{Mg} 279.553 \mathrm{~nm}, \mathrm{Mn}$ $257.611 \mathrm{~nm}$; and the tolerence level of $\mathrm{Nb}$ on $\mathrm{Mg} 279.553 \mathrm{~nm}$ and Dy $353.170 \mathrm{~nm}$ ). Hence, no contact of the organic phase will be decided based on this. It was observed that after four contacts of TOPO and TBP, the $\mathrm{Zr}$ concentration was brought down to less than $100 \mathrm{mg} \mathrm{L}^{-1}$, while for DHOA six such contacts were necessary. For $\mathrm{Ag}, \mathrm{Ba}, \mathrm{Mg}$, and $\mathrm{Mn}$, the correction factors need to be incorporated whenever necessary. The residual $\mathrm{Zr}$ after each contact of organic phase is summarized in Table V. In most cases, the spectral interference of $\mathrm{Nb}$ is very negligible as the initial amount of $\mathrm{Nb}$ in the sample is only $10 \%$.

A similar exercise was carried out for $\mathrm{Nb}$ to understand its fate after each contact of the organic phase. The initial concentration of $\mathrm{Nb}$ was kept at $1000 \mathrm{mg} \mathrm{L}^{-1}$ since only $10 \% \mathrm{Nb}$ was expected to be present. For all of these extractants, five contacts were found to bring the $\mathrm{Nb}$ concentration to below 100 $\mathrm{mg} \mathrm{L}^{-1}$. Table VI shows the residual $\mathrm{Nb}$ in the aqueous phase after each contact.

\section{Analysis of Synthetic Samples}

Synthetic samples were prepared with the composition $\mathrm{Zr}-10 \% \mathrm{Nb}$. Then the sample was processed with 1.1 $\mathrm{M}$ of ligand (TBP, DHOA, TOPO) in dodecane separately (five contacts for TBP and TOPO, but six contacts of DHOA). Then the raffinate was directly analyzed for the common 
TABLE V

The Residual Zr After Each Contact

of Organic Phase With Phase Ratio 1

\begin{tabular}{cccc}
\hline $\begin{array}{c}\text { No of } \\
\text { Contacts }\end{array}$ & $\begin{array}{c}\text { TOPO } \\
\left(\mathrm{mg} \mathrm{L}^{-1}\right)\end{array}$ & $\begin{array}{c}\text { TBP } \\
\left(\mathrm{mg} \mathrm{L}^{-1}\right)\end{array}$ & $\begin{array}{c}\text { DHOA } \\
\left(\mathrm{mg} \mathrm{L}^{-1}\right)\end{array}$ \\
\hline Initial & 10,000 & 10,000 & 10,000 \\
1 & $2500 \pm 20$ & $3333 \pm 29$ & $5555 \pm 33$ \\
2 & $555 \pm 11$ & $925 \pm 16$ & $2777 \pm 21$ \\
3 & $115 \pm 8$ & $237 \pm 9$ & $1111 \pm 13$ \\
4 & $23.1 \pm 1.7$ & $59.3 \pm 4.4$ & $444 \pm 9$ \\
5 & $4.62 \pm 0.29$ & $14.8 \pm 0.7$ & $158 \pm 8$ \\
6 & $0.92 \pm 0.03$ & $3.70 \pm 0.19$ & $56.6 \pm 3.3$ \\
7 & $0.18 \pm 0.01$ & $0.92 \pm 0.04$ & $20.2 \pm 0.8$ \\
\hline
\end{tabular}

metallic constituents. The analytical results are summarized in Table VII. This study revealed that though TOPO was found to be the most efficient extractant compared to the other two ligands, the chemical separation of the matrix using this ligand led to the loss of analytes at trace level. Therefore, TOPO cannot be used for the determination of analytes at the $1 \mathrm{mg} \mathrm{L}^{-1}$ level in a Zr-10\% Nb matrix. Both TBP and DHOA were found to be successful for the determination of $\mathrm{Ag}, \mathrm{B}, \mathrm{Ba}$, $\mathrm{Bi}, \mathrm{Ca}, \mathrm{Cd}, \mathrm{Ce}, \mathrm{Ga}, \mathrm{Gd}, \mathrm{In}, \mathrm{K}, \mathrm{La}, \mathrm{Li}$, $\mathrm{Lu}, \mathrm{Mg}, \mathrm{Mn}, \mathrm{Na}, \mathrm{Co}, \mathrm{Cr}, \mathrm{Cu}, \mathrm{Dy}, \mathrm{Eu}$, $\mathrm{Fe}, \mathrm{Nd}, \mathrm{Ni}, \mathrm{Pb}, \mathrm{Pr}, \mathrm{Sm}, \mathrm{Sr}, \mathrm{Tl}$, and $\mathrm{Zn}$ at the $1 \mathrm{mg} \mathrm{L}^{-1}$ level in $\mathrm{Zr}-10 \%$ $\mathrm{Nb}$. Since the extraction efficiency for TBP was found to be more than that of DHOA, a lesser number of contacts of organic phase was required. Therefore, five contacts of 1.1 M TBP in dodecane are considered best for preferential chemical separation of $\mathrm{Zr}$, followed by analysis of the raffinate for trace constituents.

\section{CONCLUSION}

A simple methodology was developed for the determination of $\sim 30$ analytes, including common metallic constituents and rare earth elements, at trace levels in $\mathrm{Zr}-\mathrm{Nb}$ alloy. A systematic study was car-
TABLE VI

The Residual Nb After Each Contact of Organic Phase With Phase Ratio 1

\begin{tabular}{lccc}
\hline $\begin{array}{c}\text { No of } \\
\text { Contacts }\end{array}$ & $\begin{array}{c}\text { TOPO } \\
\left(\mathrm{mg} \mathrm{L}^{-1}\right)\end{array}$ & $\begin{array}{c}\text { TBP } \\
\left(\mathrm{mg} \mathrm{L}^{-1}\right)\end{array}$ & $\begin{array}{c}\text { DHOA } \\
\left(\mathrm{mg} \mathrm{L}^{-1}\right)\end{array}$ \\
\hline Initial & 1000 & 1000 & 1000 \\
1 & 666.667 & 714.286 & 833.333 \\
2 & 416.667 & 476.19 & 555.556 \\
3 & 231.481 & 264.55 & 308.642 \\
4 & 121.832 & 125.976 & 162.443 \\
5 & 60.9162 & 59.9887 & 85.4964 \\
6 & 24.3665 & 28.5661 & 44.9981 \\
7 & 9.74659 & 13.6029 & 23.6832 \\
\hline
\end{tabular}

copy Section, Radiochemistry Division, for their constant support. lines of the elements having least spectral interference from $\mathrm{Zr}$ as well as $\mathrm{Nb}$, while still providing sufficiently good analytical performance. TBP, DHOA, and TOPO were used for optimization of the chemical separation of these major matrix elements of $\mathrm{Zr}$ and $\mathrm{Nb}$. Five contacts were found to be sufficient to bring down the $\mathrm{Zr}$ as well as $\mathrm{Nb}$ level below the tolerance level for TBP and TOPO, while six contacts of DHOA were found to be necessary to serve the purpose. Though TOPO showed the highest efficiency of $\mathrm{Zr}$ extraction, it cannot be successfully used for analytical purposes. It was observed that there was loss of a significant amount of constituents by using TOPO as the extractant. Both TBP and DHOA can be used for the trace level determination (at the $1 \mathrm{mg} \mathrm{L}^{-1}$ level) of the analytes in a $\mathrm{Zr}-\mathrm{Nb}$ matrix. Since DHOA needs more numbers of contacts which leads to the generation of more organic waste, TBP is the preferred for the present study.

\section{ACKNOWLEDGMENT}

The authors wish to acknowledge Dr. P.K. Pujari, Head, Radiochemistry Division, and Dr. R.M. Kadam, Head, Actinide Spectros-
Received November 16. 2016.

\section{REFERENCES}

1. A. Yilmazbayhan, A.T. Motta, R. J. Comstock, G. P. Sabol, B. Lai and Z. Cai, J. Nucl. Material 324 (1), 6 (2004).

2. A. T. Mott, A. Yilmazbayhan, M. J. Gomes da Silva, R. J. Comstock, G. S. Was, J. T. Busby, E. Gartner, Q. Peng, Y. H. Jeong and J. Y. Park, J. Nucl. Material 371 (1-3), 61 (2007).

3. B. Cox, J. Nucl. Material 336 (2-3), 331 (2005).

4. K.L. Murtya and I. Charit, J. Nucl. Material 383 (1-2), 189 (2008).

5. C.C. Dollins and M. Jursich, J. Nucl. Material 113 (1), 19 (1983).

6. Y. H. Jeong, S-Y Park, M-H Lee, B-K Choi, J-H Baek and J-Y Park, J. Nucl. Sci. Technol. 43 (9), 977 (2006).

7. R. Tewari, D. Srivastava, G.K. Dey, J.K. Chakravarty and S. Banerjee, J. Nucl. Material 383 (1-2), 153 (2008).

8. W.J. Langford and L.E.J. Mooder, Int. J. Press Vessl. Piping 6(4), 275 (1978).

9. D. O. Northwood, Material and Design 6 (2), 58 (1985). 
TABLE VII

Analytical Results for Synthetic Samples of Zr-10\% Nb

\begin{tabular}{lccccc}
\hline Elements & $\begin{array}{c}\text { Analytical } \\
\text { Line }(\mathrm{nm})\end{array}$ & $\begin{array}{c}\text { Initial } \\
\left(\mathrm{mg} \mathrm{L}^{-1}\right)\end{array}$ & $\begin{array}{c}\text { TBP } \\
\left(\mathrm{mg} \mathrm{L}^{-1}\right)\end{array}$ & $\begin{array}{c}\text { TOPO } \\
\left(\mathrm{mg} \mathrm{L}^{-1}\right)\end{array}$ & $\begin{array}{c}\text { DHOA } \\
\left(\mathrm{mg} \mathrm{L}^{-1}\right)\end{array}$ \\
\hline $\mathrm{Ag}$ & 338.289 & 1 & $0.95 \pm 0.04$ & $0.90 \pm 0.03$ & $0.98 \pm 0.03$ \\
$\mathrm{Al}$ & 394.401 & 1 & $0.97 \pm 0.05$ & $0.93 \pm 0.04$ & $0.97 \pm 0.04$ \\
$\mathrm{~B}$ & 182.641 & 1 & $1.03 \pm 0.04$ & $0.95 \pm 0.04$ & $0.97 \pm 0.03$ \\
$\mathrm{Ba}$ & 455.404 & 1 & $1.02 \pm 0.03$ & $0.85 \pm 0.04$ & $0.95 \pm 0.04$ \\
$\mathrm{Bi}$ & 206.170 & 1 & $0.96 \pm 0.05$ & $0.90 \pm 0.04$ & $1.03 \pm 0.05$ \\
$\mathrm{Ca}$ & 393.366 & 1 & $0.97 \pm 0.03$ & $0.87 \pm 0.04$ & $1.01 \pm 0.04$ \\
$\mathrm{Cd}$ & 226.502 & 1 & $1.05 \pm 0.05$ & $0.98 \pm 0.03$ & $0.98 \pm 0.03$ \\
$\mathrm{Ce}$ & 413.76 & 1 & $0.95 \pm 0.03$ & $0.76 \pm 0.04$ & $0.99 \pm 0.04$ \\
$\mathrm{Ga}$ & 417.206 & 1 & $0.98 \pm 0.04$ & $0.95 \pm 0.04$ & $0.96 \pm 0.05$ \\
$\mathrm{Gd}$ & 342.247 & 1 & $0.96 \pm 0.05$ & $0.79 \pm 0.04$ & $1.02 \pm 0.06$ \\
$\mathrm{In}$ & 451.131 & 1 & $0.99 \pm 0.04$ & $0.96 \pm 0.05$ & $1.03 \pm 0.05$ \\
$\mathrm{~K}$ & 766.491 & 1 & $0.99 \pm 0.03$ & $1.00 \pm 0.04$ & $1.00 \pm 0.04$ \\
$\mathrm{La}$ & 408.672 & 1 & $0.97 \pm 0.05$ & $0.82 \pm 0.05$ & $1.02 \pm 0.04$ \\
$\mathrm{Li}$ & 670.78 & 1 & $0.98 \pm 0.04$ & $0.99 \pm 0.05$ & $0.99 \pm 0.04$ \\
$\mathrm{Lu}$ & 307.76 & 1 & $0.98 \pm 0.03$ & $0.74 \pm 0.05$ & $0.97 \pm 0.05$ \\
$\mathrm{Mg}$ & 279.553 & 1 & $0.95 \pm 0.03$ & $0.95 \pm 0.05$ & $0.97 \pm 0.04$ \\
$\mathrm{Mn}$ & 257.611 & 1 & $0.95 \pm 0.04$ & $0.96 \pm 0.05$ & $0.98 \pm 0.05$ \\
$\mathrm{Na}$ & 588.995 & 1 & $0.98 \pm 0.04$ & $0.99 \pm 0.05$ & $0.98 \pm 0.05$ \\
$\mathrm{Co}$ & 228.616 & 1 & $0.98 \pm 0.03$ & $0.95 \pm 0.04$ & $0.99 \pm 0.05$ \\
$\mathrm{Cr}$ & 205.618 & 1 & $0.96 \pm 0.03$ & $0.90 \pm 0.04$ & $0.99 \pm 0.05$ \\
$\mathrm{Cu}$ & 219.958 & 1 & $0.96 \pm 0.04$ & $0.91 \pm 0.04$ & $0.99 \pm 0.05$ \\
$\mathrm{Dy}$ & 353.17 & 1 & $0.98 \pm 0.04$ & $0.77 \pm 0.04$ & $1.03 \pm 0.05$ \\
$\mathrm{Eu}$ & 390.71 & 1 & $0.95 \pm 0.04$ & $0.75 \pm 0.05$ & $1.02 \pm 0.05$ \\
$\mathrm{Fe}$ & 373.486 & 1 & $1.03 \pm 0.03$ & $0.80 \pm 0.04$ & $0.96 \pm 0.04$ \\
$\mathrm{Nb}$ & 430.358 & 1 & $1.02 \pm 0.04$ & $0.92 \pm 0.05$ & $0.98 \pm 0.05$ \\
$\mathrm{Ni}$ & 231.604 & 1 & $1.00 \pm 0.05$ & $0.97 \pm 0.04$ & $0.99 \pm 0.04$ \\
$\mathrm{~Pb}$ & 405.778 & 1 & $0.99 \pm 0.05$ & $0.96 \pm 0.05$ & $1.03 \pm 0.04$ \\
$\mathrm{Pr}$ & 417.939 & 1 & $0.99 \pm 0.02$ & $0.72 \pm 0.05$ & $1.04 \pm 0.05$ \\
$\mathrm{Sm}$ & 359.26 & 1 & $1.01 \pm 0.03$ & $0.75 \pm 0.04$ & $0.96 \pm 0.05$ \\
$\mathrm{Sr}$ & 421.556 & 1 & $1.04 \pm 0.05$ & $0.93 \pm 0.05$ & $0.98 \pm 0.05$ \\
$\mathrm{Tl}$ & 190.864 & 1 & $1.01 \pm 0.05$ & $0.95 \pm 0.04$ & $0.99 \pm 0.04$ \\
$\mathrm{Zn}$ & 213.856 & 1 & $1.02 \pm 0.05$ & $0.90 \pm 0.06$ & $0.99 \pm 0.03$ \\
\hline & & & & & \\
& & & & \\
& & &
\end{tabular}

10. M. Bojinov, V. Karastoyanova, P. Kinnunen, and T.Saario, Corrosion Science 52 (1), 54 (2010).

11. S. Banerjee and R. Krishnan, Acta Metallurgica 19 (12), 1317 (1971).

12. Y. H. Jeong, H. G. Kima, and T. H. Kim, J. Nucl. Material 317 (1), 1 (2003).

13. K.N. Chooa, Y.H. Kang, S.I. Pyun, and V.F. Urbanic, J. Nucl. Material 209 (3), 226, 1994.
18. A. Sengupta, B. Rajeswari, R.M. Kadam, and R. Acharya, At. Spectrosc 32(5), 200 (2011).

19. K. Satyanarayana and S. Durrani, J. Radioanal. Nucl. Chem. 285 (3), 659 (2010).

20. A. Sengupta, V.C. Adya, Mithelesh Kumar, S.K. Thulasidas, S.V. Godbole, and V.K. Manchanda, At. Spectrosc. 32(2), 49 (2011).

21. A. Sengupta, V. C. Adya and S. V. Godbole, J. Radioanal. Nucl Chem. 298 (2), 1117 (2013).

22. A. Sengupta, V.C.Adya, Mithlesh Kumar, S.K. Thulasidas, S.V. Godbole and V.K. Manchanda, At. Spectrosc. 32(2), 49 (2011).

23. A. Sengupta, S.K.Thulasidas and V. Natarajan, At. Spectrosc. 35(6), 247 (2014).

24. Y.Airan, A. Sengupta, S.K. Thulasidas, and V. Natarajan, At. Spectrosc. 36 (5), 221 (2015).

25. A. Sengupta, Y. Airan, S.K.Thulasidas, V.Natarajan, At. Spectrosc. 36(2), 82 (2015).

26. A. Sengupta, T. Ippili, JayabunSk, M. Singh and S.K. Thulasidas, J. Radioanal. Nucl. Chem. 310, 59 (2016).

27. A. Sengupta, M.J. Kulkarni, S.V. Godbole, V. Natarajan, and P.N. Pathak, At. Spectrosc. 35(2), 60 (2014).

28. A.Sengupta, M. J. Kulkarni and S. V. Godbole, J. Radioanal. Nucl. Chem. 289 (3), 961 (2011).

29. A. Sengupta, B. Rajeswari, R.M. Kadam, and S.V. Godbole, At. Spectrosc. 33(2), 48 (2012).

30. P.S. Somayajulu, A. Sengupta, A.K. Karande, R. Malav, D.K. Das and Mohd. Afzal, J. Radioanal. Nucl. Chem. 308(2), 495 (2016).

31. A. Sengupta, Y.Airan, S.K. Thulasidas and V. Natarajan, At. Spectrosc. 37 (2), 50 (2016).

32. K.K. Gupta, V.K. Manchanda, M.S. Subramanian, and R.K. Singh, Solv. Extr. Ion Exch. 18(2), 273 (2000). 